\title{
Modifications to the method of modal strain energy for improved estimates of loss factors for damped structures
}

\author{
Peter J. Torvik ${ }^{\mathrm{a}, *}$ and Brian Runyon ${ }^{\mathrm{b}}$ \\ ${ }^{a}$ Air Force Institute of Technology, Universal Technology Incorporated, 1866 Winchester Road, Xenia, OH 45385 , \\ USA \\ ${ }^{\mathrm{b}}$ AFRL/PRTS, Wright-Patterson AFB, OH 45433, USA
}

Received 8 May 2006

\begin{abstract}
The method of Modal Strain Energy (MSE) enables predictions of modal loss factors for vibrating systems from finite element analyses without evaluation of a complex-valued frequency response or a complex-valued frequency. While the method is simple, some error results; especially if the dissipative material has the high loss factor characteristic of materials added to increase system damping. Several methods for reducing this error through modifications to MSE have been suggested. In this work, the exact loss factor for a simple mechanical system is found. The method of Modal Strain Energy (MSE) is then used to find the loss factor for that prototype system and errors are evaluated in terms of system parameters. Comparisons are also made to predictions with several modifications to MSE. A modification due to Rongong is found to provide significant improvement. The use of this modification together with MSE is shown to lead to lower and upper bounds for the system loss factor. As the prototype system is shown to be mechanically equivalent to constrained layer damping configurations, the findings are applicable to the analysis and design of optimized sandwich beams, plates, and damping tapes. Results are given for beams and plates with constrained layer treatments.
\end{abstract}

Keywords: Damping, modal strain energy, modal loss factors

\section{Nomenclature}

$\begin{array}{ll}\mathrm{a}, \mathrm{b} & \text { Lateral dimensions of rectangular plate } \\ \mathrm{D}, \mathrm{h}, \mathrm{Y} & \text { Defined quantities: Eqs (24)-(26). } \\ \mathrm{E} & \text { Young's modulus } \\ \mathrm{E}_{E F F} & \text { Adjusted value of Young's modulus } \\ \mathrm{G} & \text { Shear modulus of damping layer } \\ \mathrm{g}^{*} & \text { Complex valued dimensionless shear parameter } \\ \Im\{\} & \text { Imaginary part of complex valued expression } \\ \mathrm{k} & \text { Stiffness of component } \\ \mathrm{K} & \text { Stiffness of system } \\ \mathrm{K}_{n m} & \text { Eigenvalue of simply supported plate } \\ \mathrm{L} & \text { Length of beam } \\ \mathrm{M} & \text { Mass of system } \\ \mathrm{m}, \mathrm{n} & \text { Mode numbers of rectangular plate }\end{array}$

${ }^{*}$ Corresponding author. Tel.: +1 937374 0521; Fax: +1 775306 0069; E-mail: Torvik@ att.net. 


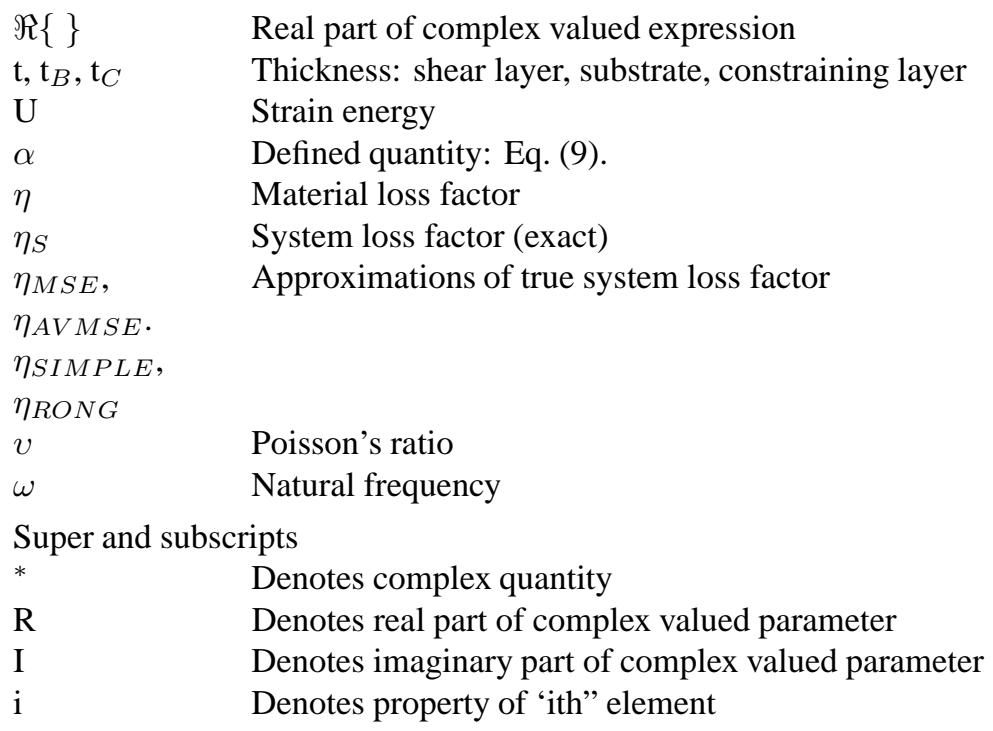

\section{Introduction}

The response of a structural system with dissipation may be predicted from a finite element analysis if the dissipative elements are represented by complex-valued moduli. By evaluating the response to harmonic input at a large number of frequencies, the modal loss factors for each mode of interest may then be determined from the computed frequency response function. Alternatively, the modal loss factors for each mode may be found from the ratio of the imaginary to real parts of the squares of the complex-valued frequencies that arise from the homogeneous solution. The method of Modal Strain Energy (MSE) [1], developed to provide a simpler and faster alternative, has proven to be satisfactory in many cases and has the further advantage of being applicable with finite element packages lacking the capacity to utilize a complex-valued modulus.

The origin of the method of Modal Strain Energy lies in an observation by Ungar and Kerwin [2] that the loss factor of a system of viscoelastic springs in parallel and/or in series may be determined from the weighted fractions of the loss factors of the individual components, with the weighting factors being the ratio of strain energy in each component to the sum of the maximum strain energies over all components. Johnson and Kienholz [1] then applied this concept to the use of finite element analysis for the determination of damping in structures containing dissipative elements. The methodology, now widely used, is generally known as the method of Modal Strain Energy (MSE). The modal loss factor for any mode of a system containing dissipative and non-dissipative portions is taken to be

$$
\eta_{S}=\frac{\text { Energy Dissipated/Cycle }}{2 \pi \text { Peak Energy Stored }}=\frac{1}{2 \pi} \frac{\sum_{\text {Diss }} 2 \pi \eta_{i} U_{i}}{\sum_{\text {Diss }} U_{i}+\sum_{\text {Non-Diss }} U_{i}}=\frac{\sum_{\text {Diss }} \eta_{i} U_{i}}{\sum_{\text {Diss }} U_{i}+\sum_{\text {Non-Diss }} U_{i}}
$$

where $\eta_{i}$ are the material loss factors for each dissipative component, $\mathrm{U}_{i}$ are the stored energies for each element in a specific mode of vibration, and $\eta_{S}$ is the system loss factor for that mode. Each dissipative component is represented by an appropriate value of Poisson's ratio and a complex-valued Young's modulus, i.e.,

$$
E^{*}=E_{R}+j E_{I}=E_{R}(1+j \eta)
$$

in which the imaginary part is proportional to the rate of energy dissipation and the real part is the customary Young's or storage modulus. The ratio of moduli is the loss factor, $\eta$, which proves to be tangent of the angle by which stress and strain are out of phase. Typically, the storage modulus of a material having high dissipative ability is strongly frequency-dependent, necessitating a preliminary estimate of the frequency of each mode of interest, and possible iteration(s) using updated values of the storage modulus. If the dissipation is dominated by one constituent of the system, and if the material loss factor of that constituent is the same in all elements (implying, in the case of 
a viscoelastic material, a uniform temperature, further simplification results and the system loss factor becomes the product of the material loss factor and a ratio of energies.

While the use of MSE is found to reduce significantly the computational effort through the elimination of the complex-valued numbers, in applications where the dissipative elements have a high material loss factor ( $\eta$ of order unity), the method of MSE has been found to over-predict the modal loss factors. Several modifications to MSE have been suggested, such as the use of MSE with the Young's modulus of the dissipative elements taken as the absolute value of the complex-valued modulus used to represent the dissipative component (AVMSE) [3], and a further improvement on that method by Rongong [4].

Elementary mechanical models consisting of spring-mass-damper systems are the fundamental units of the analysis of vibratory systems. As the finite element method may be viewed as the representation of a large assembly of such units, these systems are logical prototypes for use in evaluating the effectiveness of numerical methods in the determination of the dynamic response of mechanical systems. Rongong [4] used a two-degree-of-freedom springmass system having one dissipative element with unit loss factor to demonstrate that his suggested improvement to MSE led to better agreement with exact values over a range of a stiffness ratio.

Morgenthaler [3] used the response of a three-degree-of-freedom spring-mass system with one dissipative element (with $0<\eta<1.6$ ) as a basis for comparing the predictions from MSE and AVMSE with the exact solution. For a single set of stiffness ratios, the prediction of the system loss factor for the fundamental mode with AVMSE was found to provide improvement over that found by the use of the traditional MSE. However, McDaniel and Ginsberg [5] considered a two-degree-of-freedom spring mass system with one dissipative element (with $0<\eta<$ 1) to compare the predictions of MSE and AVMSE with the exact solution for a range of stiffness ratio, and found that the use of AVMSE with a two-degree-of-freedom system led to improved predictions for the second mode, but to larger errors for the first.

In addition to discretization errors inherent to any FEA analysis, as well as the computational challenge presented by a frequency-dependent modulus, the use of MSE introduces a third source of potential error, that being the treatment of the dissipatative material as elastic in the determination of the stored energies to be substituted into Eq. (1). The present work will focus on this issue. We will take the complex-valued modulus used to represent the damping material as a given constant and focus attention on a simple system for which an exact solution is easily obtained. Thus, any differences found between system loss factors obtained with different methods may be taken to be a consequence of the influence of the imaginary part of the modulus on the division of energy among the components of the system. The prototype system chosen for the analysis consists of two elastic and one dissipative spring element with $\eta=1$. System loss factors as found by various approximate methods are compared with exact values over ranges of the two stiffness ratios resulting after normalizing by the stiffness of one of the elastic elements.

The application of MSE to the evaluation of constrained layer damping treatments and sandwich beams and plates presents a particular challenge when materials with high loss factors are used. Although such materials typically have inherently low moduli, the application in thin layers creates a high effective stiffness that has a significant influence on the distribution of energy within the structure, thereby impacting the accuracy of the method of MSE. It will be shown that, in the case of simply-supported ends, sandwich beams with dissipative cores and beams with constrained layer treatments (damping tapes) are mechanically equivalent to the chosen prototype four parameter mechanical system. Predicted loss factors found through various modifications of MSE will be compared to those obtained from the complex valued response as found by finite element methods and also to exact solutions for system parameters characteristic of beams optimized for maximum damping.

\section{An elementary model of a dissipative system}

The four parameter system of Fig. 1 is taken as a prototype. Springs $k_{1}$ and $k_{3}$ are elastic and spring $k_{2}=k^{*}$ (of complex material modulus $E^{*}$ ) is dissipative. This system is equivalent to the standard linear anelastic solid [6] with the viscous component replaced by a structural damping element, i.e., an element represented by a complexvalued modulus. The resulting system, now having a complex-valued stiffness, $K^{*}$, when attached to a mass, forms the single degree of freedom system chosen by Marsh and Hale [7] as being characteristic of damped beam-like structures. 


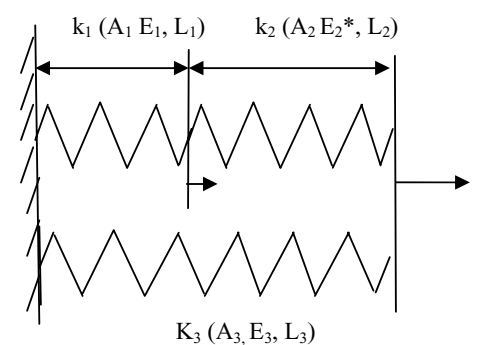

Fig. 1. A four parameter prototype system.

The stiffness of each element is related to a modulus, length, and area through $k_{i}=A_{i} E_{i} / L_{i}$. Thus, if a spring is made of a material with represented by a complex-valued modulus, $E^{*}=E_{R}(1+j \eta)$, the stiffness $k^{*}$ has a complex value, $k^{*}=k_{R}(1+j \eta)$, with the same loss factor, where the symbol $\eta$ (without subscript) represents a material loss factor and the subscript ' $R$ ' denotes the real part of a complex quantity The representation of the assembly with a complex-valued stiffness is easily obtained by the viscoelastic correspondence principle [8], i.e., first treating all springs as elastic, $k^{*} \Rightarrow k_{2}$, finding the real-valued system stiffness, and then setting $k_{2} \Rightarrow k^{*}$, to yield a complex-valued system stiffness:

$$
K^{*}=\frac{1}{1 / k_{1}+1 / k^{*}}+k_{3}=K_{R}\left(1+j \eta_{S}\right)
$$

The system loss factor, $\eta_{S}$, is given by the ratio of imaginary and real parts of the system stiffness and can be expressed in terms of the material loss factor, $\eta$, and the two stiffness ratios, $k_{R} / k_{1}$ and $k_{3} / k_{R}$.

$$
\eta_{S}=\frac{\eta}{\left[1+\frac{k_{R}}{k_{1}}\left(1+\eta^{2}\right)\right]+\frac{k_{3}}{k_{R}}\left\{\left(1+\frac{k_{R}}{k_{1}}\right)^{2}+\left(\eta \frac{k_{R}}{k_{1}}\right)^{2}\right\}}
$$

If a mass $M$ is added to the right hand side of the system of Fig. 1, the natural frequency $\left(\omega_{n}\right)$ may be found from the homogeneous solution. With a complex-valued stiffness, the natural frequency is complex, with the imaginary part giving the rate of decay of oscillation. In a forced motion, resonance occurs at the coincidence of excitation frequency with the real part,

$$
\Re\{\omega\}=\sqrt{\frac{\left|K^{*}\right|}{M}} \cos \left[\left(\arctan \eta_{S}\right) / 2\right]
$$

\section{Modal Strain Energy}

For the system of Fig. 1, the only dissipative element is element 2. Elements 1 and 3 are taken as non-dissipative. In the traditional application of MSE, the real parts of complex-valued modulus of the dissipative elements are used as a real modulus in the evaluation of system energies. When $k^{*}$ is taken as $k_{2}$, these energies are easily found for the system of Fig. 1. The loss factor is then found from Eq. (1) to be

$$
\eta_{M S E}=\frac{\eta}{1+\frac{k_{2}}{k_{1}}+\frac{k_{3}}{k_{2}}\left(1+\frac{k_{2}}{k_{1}}\right)^{2}}
$$

Also,

$$
\omega_{M S E}=\sqrt{\frac{K_{E F F}}{M}}=\sqrt{\frac{1}{M}\left[k_{3}+\frac{1}{1 / k_{2}+1 / k_{1}}\right]}
$$

The loss factor as obtained by MSE can then be compared with the true value. We find that the error resulting from the use of modal strain energy to be related to the material loss factor and the stiffness ratios through: 


$$
\frac{\Delta \eta_{S Y S}}{\eta_{S}}=\frac{\eta_{M S E}-\eta_{S}}{\eta_{S}}=\eta^{2}\left\{\frac{k_{R}}{k_{1}}+\frac{k_{3}}{k_{R}}\left(\frac{k_{R}}{k_{1}}\right)^{2}\right\} /\left\{1+\frac{k_{R}}{k_{1}}+\frac{k_{3}}{k_{R}}\left(1+\frac{k_{R}}{k_{1}}\right)^{2}\right\}
$$

The error is seen to be positive for any combination of parameters appearing in the system of Fig. 1, confirming the well known empirical observation that the method of MSE will over-predict the system loss factor. It is also seen that the error increases with the square of the material loss factor and goes to zero as $S=k_{R} / k_{1} \Rightarrow 0$ for any choice of $\eta$ and $k_{3} / k_{R}$. However, a low system loss factor does not assure an accurate evaluation by MSE. If the ratio $k_{3} / k_{R}$ is large, the system loss factor of Eq. (4) is necessarily low. But the error, from Eq. (8) with $\eta$ of order unity and large $k_{3}$, is between $25 \%$ and $100 \%$ for any stiffness ratio $S=k_{R} / k_{1}>0.8$.

One might then ask if there is any simple relationship that might be used to select an effective stiffness of the form $k_{2}=f\left(k_{R}, \eta\right)$ for use in MSE such that equality is achieved in the determination of loss factors by Eqs (4) and (6). This does not appear to be possible. It may be shown, however, that in the limit as either $k_{1}$ or $k_{3} \Rightarrow 0$ the use of an effective stiffness $k_{2}=k_{R}\left(1+\eta^{2}\right)$ in MSE does lead to the correct loss factors.

\section{Modifications to method of Modal Strain Energy}

\subsection{Absolute Value Modal Strain Energy (AVMSE)}

It has been suggested [3] that the method of Modal Strain Energy (MSE) be applied with the finite element calculations performed by using the magnitude of the complex-valued modulus of the dissipative elements as the real values of modulus in the finite element analysis. Application of this methodology (AVMSE) to the system of Fig. 1 leads to the loss factor and frequencies of Eqs (6) and (7), with the real stiffness $k_{2}$ replaced by $\alpha k_{R}$, where

$$
\alpha=\sqrt{1+\eta^{2}}
$$

The resulting estimate of the system loss factor is

$$
\eta_{A V M S E}=\frac{\eta}{1+\frac{\alpha k_{R}}{k_{1}}+\frac{k_{3}}{\alpha k_{R}}\left(1+\frac{\alpha k_{R}}{k_{1}}\right)^{2}}
$$

and the new estimate of the natural frequency is

$$
\omega_{A V M S E}=\sqrt{\frac{K_{E F F}}{M}}=\sqrt{\frac{1}{M}\left[k_{3}+\frac{1}{1 / \alpha k_{R}+1 / k_{1}}\right]}
$$

Since the factor, $\alpha$, appears in both the numerator and denominator of the denominator of the expression for the loss factor, and is always greater than one, it can be expected that the use of AVMSE will lead to higher system loss factors than traditional MSE in some cases, and lower in others, depending on the other system parameters. However, the frequency obtained with AVMSE will always be higher than that found with MSE.

As before, the error in the resulting loss factor may be evaluated by comparing Eq. (10) with Eq. (4). The fractional difference is:

$$
\frac{\Delta \eta_{S Y S}}{\eta_{S}}=\frac{\eta_{A V M S E}-\eta_{S}}{\eta_{S}}=(\alpha-1)\left\{\frac{k_{3}}{\alpha k_{R}}+\frac{\alpha k_{R}}{k_{1}}\left(1+\frac{k_{3}}{k_{1}}\right)\right\} /\left\{1+\frac{\alpha k_{R}}{k_{1}}+\frac{k_{3}}{\alpha k_{R}}\left(1+\frac{\alpha k_{R}}{k_{1}}\right)^{2}\right\}
$$

Evaluations of the limiting cases of small $k_{1} / k_{R}$, large $k_{1} / k_{R}$, small $k_{3} / k_{R}$, and large $k_{3} / k_{R}$ show that the error goes to zero only when $\eta \Rightarrow 0$.

Errors resulting from the determination of the system loss factor by AVMSE are compared in Fig. 2 with the errors resulting from the use of traditional MSE (Eq. (8)) for the case of $\eta=1$, i.e. $\alpha=\sqrt{ } 2$, and various values of stiffness ratios $k \equiv k_{1} / k_{3}$ and $S=k_{R} / k_{1}$. Errors with AVMSE are seen to range from nearly $20 \%$ to about $40 \%$ for the range considered, and to be greater than the errors resulting from the use of modal strain energy unless the stiffness ratio, $S=k_{R} / k_{1}$ is greater than (approximately) 0.5 . 


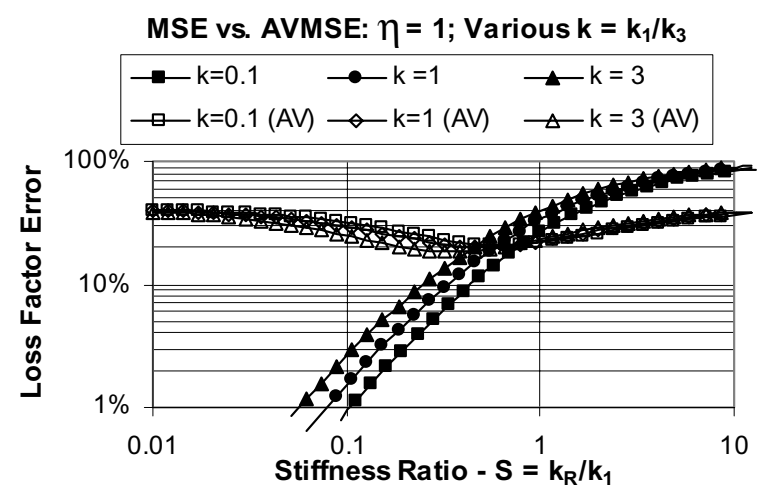

Fig. 2. Comparison of error: MSE vs. AVMSE modification.

\subsection{Rongong's modification}

If one seeks to use MSE with an effective stiffness $k_{2}$ (obtained by replacing $E$ with a real-valued effective modulus $\left.E_{E F F}\right)$ it is appropriate to consider the consequences in the evaluation of the dissipated and strain energies. The resulting strain energy in the dissipative element 2 of Fig. 1, when written in terms of the concomitant effective strains is

$$
U_{2}=\int_{V o l} E_{E F F} \frac{\varepsilon_{E F F}^{2}}{2} d v o l
$$

In the application of MSE, the dissipated energy is taken as

$$
D_{2}=2 \pi \eta U_{2}=2 \pi \eta \int_{V o l} E_{E F F} \frac{\varepsilon_{E F F}^{2}}{2} d v o l
$$

But a more appropriate value for the dissipated energy would be that found from the loss modulus, or imaginary part, $E_{I},=\eta E_{R}$ of the complex-valued modulus of the dissipative material [9]. The dissipated energy is then

$$
D_{2}=\pi \int_{V o l} E_{I} \varepsilon_{E F F}^{2} d v o l=2 \pi \frac{E_{I}}{E_{R}} \frac{E_{R}}{E_{E F F}} \int_{V o l} E_{E F F} \frac{\varepsilon_{E F F}^{2}}{2} d v o l=2 \pi \eta \frac{E_{R}}{E_{E F F}} U_{2}
$$

Thus, the energy dissipated should be reduced (for use in MSE) by the ratio $E_{R} / E_{E F F}=k_{R} / k_{2}$. This may be achieved either by replacing the material loss factor for each dissipative element, $\eta_{i}$, in Eq. (1) by an effective loss factor,

$$
\eta_{E F F}=\eta \frac{E_{R}}{E_{E F F}}
$$

or by replacing $U_{2}$ in the same equation by an effective energy, computed from the energy found by Eq. (13) as

$$
\hat{U}=\frac{E_{R}}{E_{E F F}} U_{2}
$$

Rongong [4] took the second approach and modified the method of MSE by using the magnitude of the complexvalued modulus as the effective modulus in MSE as was done in AVMSE. The equivalent in the prototype system is that:

$$
k_{2}=\alpha k_{R}=k_{R} \sqrt{1+\eta^{2}}
$$

But when the effective energy of Eq. (17) is then used in Eq. (1), the system loss factor is modified to

$$
\eta_{R O N G}=\frac{\eta \hat{U}_{2}}{U_{1}+\hat{U}_{2}+U_{3}}=\frac{\eta U_{2} / \alpha}{U_{1}+U_{2} / \alpha+U_{3}}
$$




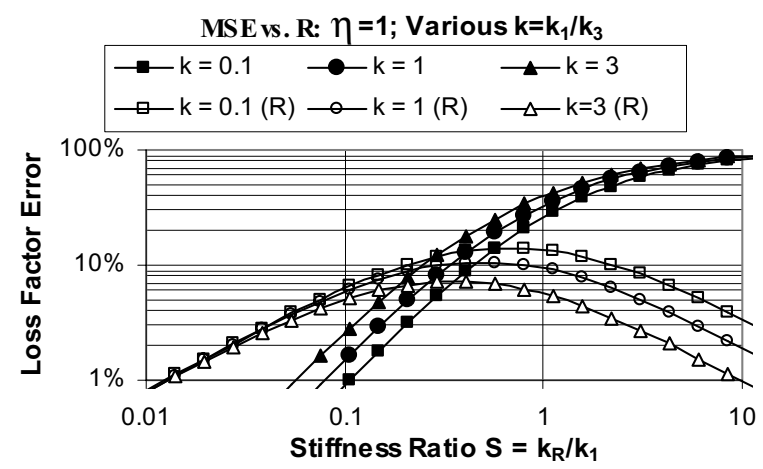

Fig. 3. Comparison of error: MSE vs. Rongong's modification.

For the system of Fig. 1, the loss factor obtained by using Eq. (18) with Eq. (19) then becomes

$$
\eta_{R O N G}=\frac{\eta}{1+\frac{\alpha^{2} k_{R}}{k_{1}}+\frac{k_{3}}{k_{R}}\left(1+\frac{\alpha k_{R}}{k_{1}}\right)^{2}}
$$

The error resulting from the use of Eq. (20) may be compared with $\eta_{S}$, the exact value from Eq. (4). The fractional difference is

$$
\frac{\eta_{S}-\eta_{R O N G}}{\eta_{S}}=-\frac{k_{3}}{k_{1}} \frac{2(\alpha-1)}{1+\frac{\alpha k_{R}}{k_{1}}+\frac{k_{3}}{k_{R}}\left(1+\frac{\alpha k_{R}}{k_{1}}\right)^{2}}
$$

The error, which is always negative, goes to zero as $\eta \Rightarrow 0$ for $k_{3} / k_{1} \Rightarrow 0$, for $k_{R} / k_{1} \Rightarrow 0$, or for $k_{R} / k_{1} \Rightarrow \infty$. For small values of $k_{1} / k_{3}$ and $\eta=1$, the maximum error remains at about $14 \%$.

The fractional errors resulting from the use of Eq. (20) may be compared with the exact value and the error resulting from traditional MSE, Eq. (8). A comparison for $\eta=1(\alpha=\sqrt{ } 2)$ and various stiffness ratios $k=k_{1} / k_{3}$ and $S=k_{R} / k_{1}$ is given in Fig. 3 .

For small values of the stiffness ratio $k_{R} / k_{1}$ the unmodified MSE again provides superior predictions, but even in this case the errors resulting from the use of the Rongong modification are modest.

An estimate of frequency may also be found. Since the squared frequency is proportional to stored energy, a frequency consistent with Rongong's estimation of the loss factor may be found by reducing the frequency resulting from the use of AVMSE by the ratio of stored energies, i.e.

$$
\begin{aligned}
\omega_{R} & =\omega_{A V M S E} \sqrt{\frac{U_{1}+U_{2} / \alpha+U_{3}}{U_{1}+U_{2}+U_{3}}} \\
& =\omega_{A V M S E}\left[\frac{k_{1}}{\alpha^{2} k_{2}}+\left\{1+\frac{k_{3}}{k_{1}}\left(1+\frac{k_{1}}{\alpha k_{2}}\right)^{2}\right\}\right]^{1 / 2} /\left[\frac{k_{1}}{\alpha k_{2}}+\left\{1+\frac{k_{3}}{k_{1}}\left(1+\frac{k_{1}}{\alpha k_{2}}\right)^{2}\right\}\right]^{1 / 2}
\end{aligned}
$$

\subsection{A simple correction}

The necessary correction to the dissipated energy, Eq. (15), could also have been made through adjusting the loss factor, as in Eq. (16). In this case, we find a simple correction to the method of MSE. The resulting loss factor would be simply:

$$
\eta_{S I M P L E}=\frac{\eta U_{2} / \alpha}{U_{1}+U_{2}+U_{3}}=\frac{\eta_{A V M S E}}{\alpha}
$$

The frequency consistent with this approximation is the same as that found by AVMSE. 


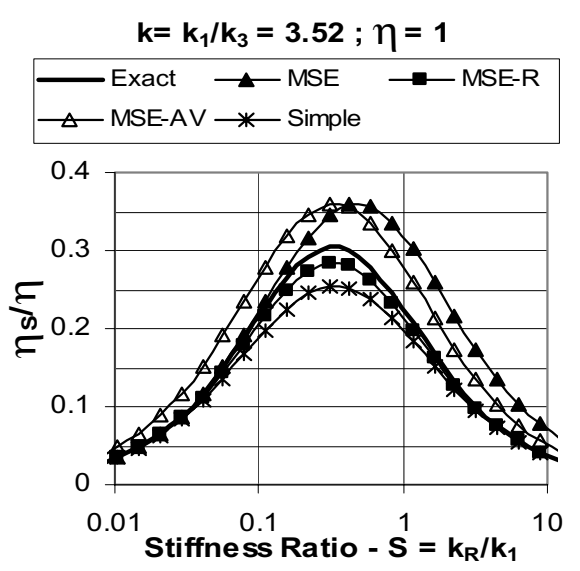

a. Typical Sandwich Beam Configuration

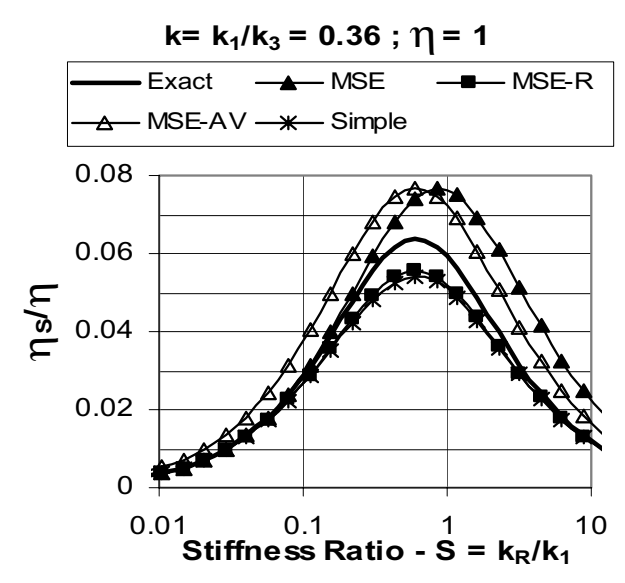

b. Typical Damping Tape Application

Fig. 4. Loss factors predicted with modification to Modal Strain Energy (a. Typical sandwich beam configuration) (b. Typical damping tape application).

\subsection{Comparisons}

It will be shown in a later section that a stiffness ratio of $k=k_{1} / k_{3}=3.52$ is representative of a sandwich configuration with thin core, and that $k=0.36$ is representative of a damping tape configuration. Figure 4 shows the exact value of the system loss factor as given by Eq. (4) and the value found by the traditional application of MSE, Eq. (6) for these values of stiffness and a material loss factor of $\eta=1$. Also shown are values obtained through several modifications. Results obtained with Rongong's modification, Eq. (20), are coded MSE-R; results obtained by using the absolute value of the complex-valued modulus in MSE, Eq. (10), are labeled MSE-AV; and results obtained by simply dividing the AVMSE result by the parameter $\alpha$, Eq. (23), are coded Simple.

Although occurring at different values of the stiffness ratio, $S$, it is of interest to note that MSE and AVMSE both predict the same maximum values of the system loss factor, and that this remains true for significantly different values of the stiffness ratio, $k$. The value predicted by both, however, is significantly higher than the true value. MSE is seen to provide decidedly superior predictions for systems with low values of stiffness $S=k_{R} / k_{1}$, while the absolute value modification is seen to provide improved results for systems with high values of $S$. It would appear that this dependence on stiffness accounts for the apparent contradictions seen in the comparisons made in [3,5]. While MSE and AVMSE are seen to lead to overestimates of the loss factor, the use of Rongong's modification or the simpler modification to AVSME, appear to lead to under-predictions.

An interesting and important attribute of the various modifications to the method of MSE is the correctness to which the configuration for maximum damping is identified. From Fig. 4 we may note that each of the modifications considered appears to predict the same required value of $S=k_{R} / k_{1}$ as does the exact solution, whereas the traditional method of MSE leads to a higher value.

The errors resulting from the use of several modifications to the method of MSE are compared in Fig. 5. Shown in each case is the difference between the predicted value and the true value, Eq. (4), expressed as a percent of the true value. All curves are labeled as in Fig. 4. The unaltered MSE is seen to provide the best predictions for values of stiffness $S<0.1$ for configurations typical of sandwich plates or for values of stiffness $S<0.45$ for configurations typical of damping tapes. Note that all comparisons given here are for the case of a material loss factor $(\eta)$ of unity.

Because MSE provides an underestimate of the stored energy, it also gives rise to underestimates of natural frequencies. Similarly, the overestimate of stored energy associated with AVMSE leads to overestimates of frequencies. Errors in frequency for various stiffness ratios may be evaluated by comparing the predictions of Eqs (7), (11), and (22) with the exact values as given by Eq. (5). Some consideration of such frequency estimates are given elsewhere [10]. An empirical relationship of the form $k_{2}=k_{R} f\left(\eta, k_{R} / k_{1}\right)$ has been found [11] to give quite satisfactory estimates of frequency and, when used as the stiffness of the dissipative element in MSE, quite good estimates of loss factors. 


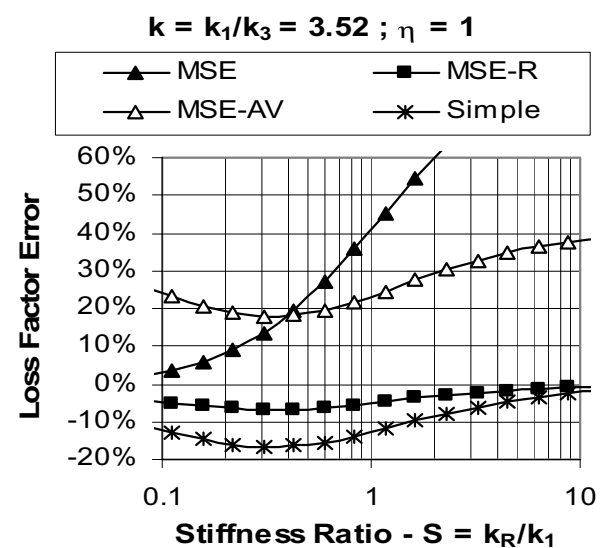

a. Typical Sandwich Beam Configuration

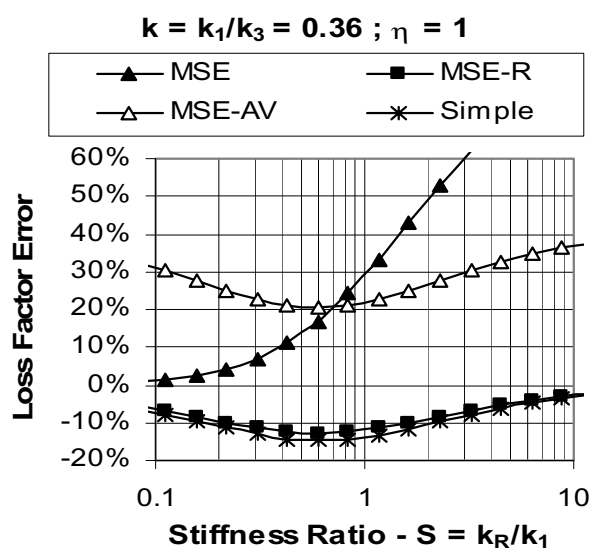

b. Typical Damping Tape Application

Fig. 5. Errors with modifications to MSE (approximate-true/true) (a. Typical sandwich beam configuration) (b. Typical damping tape application).

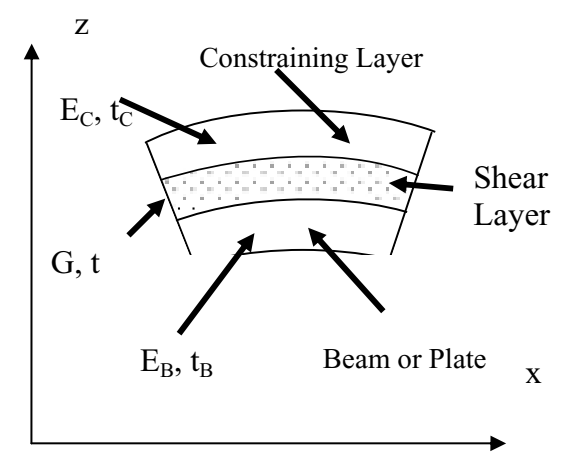

Fig. 6. The constrained layer configuration.

Because the errors are small, the optimal configuration is correctly identified, and the estimates of damping are always conservative; the modification to MSE suggested by Rongong appears to have the greatest merit. However, it is to be noted that the use of MSE together with the Rongong approximation appears to provide a quick and reliable means of obtaining upper and lower bounds on system loss factors.

\section{Beams and plates with constrained layer damping}

While extensions to such multi-dimensional structures as rings, and shells are found in the literature, we consider here only beams and thin plates with damping enhancement through the use of a thin dissipative layer undergoing shear deformation. The constraining layer shown in Fig. 6 may be comparable to the thickness of the beam or plate, or much thinner, as in a damping tape application.

We accept here the customary assumptions and concomitant limitations, namely that: longitudinal inertia is negligible, shear deformations in the beam and constraining layer are negligible, the thickness of the shear layer remains constant, strain energies due to extensions in the shear layer are negligible, shear strain is uniform through the thickness of the shear layer, and all bonds are perfect.

In the very special case of the simply-supported beam of length $L$, fully covered on one side with a constraining layer and vibrating in the nth mode, a complex-valued stiffness may be identified from the analysis of the constrained layer damping treatment as given by Ross et al. [12]. Let:

$$
D=\left(E_{B} t_{B}^{3}+E_{C} t_{C}^{3}\right) / 12
$$




$$
\begin{aligned}
h & =1+\left(t_{B}+t_{C}\right) / 2 t \\
Y & =\frac{(t h)^{2}}{D}\left(\frac{1}{E_{B} t_{B}}+\frac{1}{E_{C} t_{C}}\right)^{-1} \\
g^{*} & =\frac{G^{*}}{t}\left(\frac{1}{E_{B} t_{B}}+\frac{1}{E_{C} t_{C}}\right)\left(\frac{L}{n \pi}\right)^{2} \equiv g_{R}(1+j \eta)
\end{aligned}
$$

The complex bending stiffness is found to be

$$
D^{*}=D\left[1+Y \frac{g^{*}}{1+g^{*}}\right]
$$

In the above, $E_{B}, E_{C}, t_{B}, t_{C}$ are the moduli and thickness of beam and constraining layer, respectively. The thickness and complex-valued modulus of the shear layer are $t$ and $G^{*}=G_{R}(1+j \eta)$. When used as a damping addition (i.e., damping tape) the constraining layer stiffness is typically much less than that of the beam $\left(E_{C} t_{C} \ll E_{B} t_{B}\right)$, while for the symmetric sandwich beam $E_{C} t_{C} \equiv E_{B} t_{B}$. The system loss factor is then found from the ratio of imaginary and real parts of the effective system stiffness to be

$$
\eta_{S}=\frac{\Im\left\{D^{*}\right\}}{\Re\left\{D^{*}\right\}}=\frac{\eta Y g_{R}}{1+(2+Y) g_{R}+(1+Y)\left(1+\eta^{2}\right) g_{R}^{2}}
$$

A method for determining the loss factor for beams of very general boundary conditions has been developed by Rao [13], but a numerical solution is required and results have been obtained only for selected values of parameters. Abdulhadi [14] developed the equations of motion for the three layer plate. It has been shown [15] that, in the case of the a x b plate simply supported on all edges, with constraining layer of the same Poisson's ratio, $v$, and free edges, that the resulting loss factor is that given above if the squared eigenvalue for the beam in Eq. (27) is simply replaced by

$$
\left(\frac{L}{n \pi}\right)^{2}=\frac{1}{1-v^{2}} \frac{1}{K_{n m}^{2}}=\frac{1}{1-v^{2}}\left\{\left(\frac{a}{n \pi}\right)^{2}+\left(\frac{b}{m \pi}\right)^{2}\right\}
$$

Approximations for other boundary conditions may be obtained by replacing the eigenvalue, $\mathrm{K}_{n m}$, by that of the plate [15] or beam [16] of interest. In the case of beams, $v$ is set to zero.

Plunkett and Lee [17] have shown that the maximization of the loss factor for a constrained layer treatment requires a specific combination of parameters. That combination may be identified by differentiating the expression for the loss factor, Eq. (29) with respect to $g_{R}$ and setting the result to zero. The maximum loss factor is found to occur for

$$
(1+Y)\left(1+\eta^{2}\right) g_{R}^{2}=(1+Y)\left|g^{*}\right|^{2}=1
$$

\section{Equivalence of prototype and constrained layer systems}

Manipulation of Eq. (28) gives for the constrained layer on a beam that

$$
D^{*}=D\left[1+\frac{Y g^{*}}{1+g^{*}}\right]=D+\frac{1}{1 /(D Y)+1 /\left(D Y g^{*}\right)}
$$

and a comparison with the complex-valued stiffness of the prototype system, Eq. (3), shows that the functional forms are identical. Thus, the constrained layer treatment is mechanically equivalent to the system depicted in Fig. 1 and the parameters of the constrained layer treatment, Eqs (24)-(28), may be related to the effective spring constants $k_{1}, k^{*}, k_{R}$, and $k_{3}$. The result is that the constrained layer treatment in the case of pinned ends may be precisely represented by the prototype system with stiffness parameters

$$
k_{3}=D_{=} \frac{1}{12}\left\{E_{B} t_{B}^{3}+E_{C} t_{C}^{3}\right\}
$$




$$
\begin{aligned}
& k_{1}=D Y=(t h)^{2}\left(\frac{1}{E_{C} t_{C}}+\frac{1}{E_{B} t_{B}}\right)^{-1} \\
& k^{*}=D Y g^{*}=t h^{2} G^{*} L^{2} / \pi^{2}
\end{aligned}
$$

Using these, the modifications to the method of MSE as were considered above may be applied directly to the analysis of constrained layer treatments.

The parameter $k_{3}$ is recognized as the effective stiffness of two parallel, but uncoupled beams. The quantity $k_{1}=D Y$ may be recognized from Eq. (28) as the increase in system stiffness that results if the two beams are fully coupled by a stiff shear layer, i.e. with $g^{*} \Rightarrow \infty$. This occurs if the shear layer thickness is taken to zero and the perfect bond is maintained. The remaining term, $k^{*}$, is not easily provided with a physical interpretation, other than to note that it is the only contribution of shear layer modulus to the system stiffness and that the loss factor in the representation of $k^{*}$ is the loss factor of the shear layer material.

The stiffness ratios of Eq. (4) may be evaluated from Eqs (33)-(35). For the symmetric sandwich, $t_{C}=t_{B}$, and:

$$
\frac{k_{1}}{k_{3}}=Y=\frac{(t h)^{2}}{D}\left(\frac{1}{E_{B} t_{B}}+\frac{1}{E_{C} t_{C}}\right)^{-1}=\frac{(t h)^{2} E_{B} t_{B} / 2}{2\left(E_{B} t_{B}^{3}\right) / 12}=3\left[1+\frac{t}{t_{B}}\right]^{2}
$$

or approximately 3 if $t / t_{B} \ll 1$. For a beam much thicker than either constraining layer or shear layer

$$
\frac{k_{1}}{k_{3}}=Y=\frac{(t h)^{2}}{D}\left(\frac{1}{E_{B} t_{B}}+\frac{1}{E_{C} t_{C}}\right)^{-1} \cong \frac{\left(t_{b} / 2\right)^{2} E_{C} t_{C}}{E_{B} t_{B}^{3} / 12}=3 \frac{E_{C} t_{C}}{E_{B} t_{B}}
$$

which goes to zero as $t_{C} / t_{B} \Rightarrow 0$.

In either case, the stiffness ratio $k^{*} / k_{1}$ proves to be the complex shear parameter

$$
\frac{k_{R}}{k_{1}}(1+j \eta)=\frac{k^{*}}{k_{1}}=\frac{D Y g^{*}}{D Y}=g_{R}(1+j \eta)
$$

and the condition for maximum damping as given by Eq. (31) is

$$
\left|g^{*}\right|_{\text {MAX }}=\frac{1}{\sqrt{(1+Y)}}
$$

For the special case of the symmetric simply supported sandwich beam or plate of core of thickness $t \ll t_{B}$, maximum damping requires $\left|g^{*}\right|_{M A X} \cong 1 / 2$. For the case of $t_{B} \gg t_{C}$ and $t_{B} \gg t$, maximum damping requires $\left|g^{*}\right|_{\text {MAX }} \cong 1$. For any configuration the combination of parameters required for maximum damping is given by:

$$
\left.\left[\frac{G_{R}}{t\left(1-v^{2}\right)}\left(\frac{1}{E_{B} t_{B}}+\frac{1}{E_{C} t_{C}}\right)\left\{\left(\frac{a}{n \pi}\right)^{2}+\left(\frac{b}{m \pi}\right)^{2}\right\}\right]_{M A X} \equiv g_{R}\right|_{M A X}=\frac{1}{\sqrt{(1+Y)\left(1+\eta^{2}\right)}}
$$

In the case of the simply supported beam, $b=0, v=0$ and $a=L$. For $t_{C}=t_{B}=12 t, k_{1} / k_{3}=Y=3.52$ and maximum damping occurs, for $\eta=1$, at $k_{R} / k_{1}=\left.g_{R}\right|_{M A X}=0.332$. For $t_{C}=t=t_{B} / 12$ and $E_{C}=E_{B}$, $k_{1} / k_{3}=Y=0.36$ and maximum damping (for $\eta=1$ ) occurs at $k_{R} / k_{1}=\left.g_{R}\right|_{\text {MAX }}=0.606$. These are seen to be the location of the maximum values of system loss factors in Fig. 4, which were specifically computed for these parameters.

\section{Comparison of results}

Soni and Bogner [18] used Rao's sixth-order theory results to compute loss factors and frequencies for a $177.8 \mathrm{~mm}$ (7 in.) long, $25.4 \mathrm{~mm}$ ( 1 in.) wide, symmetric sandwich beam of aluminum $(E=69 \mathrm{GPa}$ or $10 \mathrm{Mpsi}, v=0.3$, and $\rho=2.8 \mathrm{gm} / \mathrm{cc})$ with facing sheets $1.524 \mathrm{~mm}(0.060 \mathrm{in})$ thick. The $0.127 \mathrm{~mm}(0.005 \mathrm{in})$ thick viscoelastic core had as other properties $E=2.1 \mathrm{MPa}$ or $300 \mathrm{psi}, v=0.499$ or $G=100 \mathrm{psi}, \eta=1$, and $\rho=0.97 \mathrm{gm} / \mathrm{cc}$. Their results were then compared with findings from a finite element computation. Loss factors were extracted from the real part of a FEA computed frequency and showed excellent agreement with the predictions of the "exact" sixth-order theory. 
As the symmetric sandwich cantilever used in these computations has been used by several other investigators, it is particularly well characterized and suited for use in comparison with loss factors obtained by MSE and other approximate methods. Loss factors for the first six modes of the damped sandwich cantilever beam having these properties were determined with FEA using ANSYS by the method of Modal Strain Energy (MSE); MSE using the absolute value of the shear layer modulus (AVMSE); MSE with the modification due to Rongong; and with the simple adjustment obtained from dividing the loss factors obtained with AVMSE by $(1+\eta)^{1 / 2}$. Loss factors were also computed from complex eigenvalues found using a complex-valued modulus in Nastran.

ANSYS 8.1 was used to obtain loss factors and frequencies by MSE. Nastran 2004 was used to obtain loss factors and frequencies from the complex valued eigenvalues. In both cases the elements used were three-dimensional, 8-noded solid brick elements. Each node had three translational degrees of freedom. These elements are SOLID45 bricks in ANSYS and CHEXA elements in Nastran. In order to provide the same mesh density in both directions for all aspect ratios, square elements were chosen. This allowed an integer number of elements in both directions for all aspect ratios $(0.5,1,2,3.5,7$, and 14) considered. A frequency convergence study for a 7 " square cantilevered plate showed a $42 \times 42$ mesh to be adequate. Thus, square elements of 1/6 inch side length were used in all cases. Results were then compared for several modes of the 7 inch square cantilever plate with the same mesh and with 1 , 2 , and 3 elements through each of the three layers. As loss factors agreed to within $0.4 \%$ (with $90 \%$ of the variation occurring in going from one to two elements) and the variations in frequency were similar, one element through the thickness of each layer was accepted as sufficient. The total number of elements varied with aspect ratio, from 378 at $A R=14$ to 10584 at $A R=1 / 2$. As one element was taken through the thickness of each layer, the element aspect ratio in the shear and constraining layers was 33.3, and less than 3 in the substrate. In the case of a free or simply supported edge, both components of the in-plane displacement were allowed to develop freely. In the case of a clamped edge, both components were fully restrained.

Loss factors by AVMSE $\left(\eta_{\alpha}\right)$ were computed using MSE with the modulus of the shear layer taken as $G_{=} \alpha G_{R}$, with $\alpha=(1+\eta)^{1 / 2}$. The loss factors with the Rongong modification then may be shown from Eq. (19) to be simply

$$
\frac{\eta_{R}}{\eta}=\frac{\eta_{A V M S E} / \eta}{\alpha+(1-\alpha) \eta_{A V M S E} / \eta}
$$

and those for the simple modification result from Eq. (23).

The results are compared in Fig. 7. Loss factors determined from complex frequencies (FEA) were in satisfactory agreement with those obtained by Soni and Bogner, and also those obtained by Sun et al. [19] for the same configuration. Small differences between the FEA and analytic results are presumed to be due to the fact that the FEA allows more complex deformations than the idealizations of the Ross, Ungar, Kerwin formulation that are incorporated in the Rao analysis.

The expected consistent over-predictions with MSE are evident. Some improvement is provided by AVMSE at the lowest mode number, corresponding to long wavelengths and high values of $g_{R}=k_{R} / k_{1}$. See Eq. (38) and Fig. 4(a). But this is achieved at the expense of notable error at the higher mode numbers. The results using both the modification due to Rongong (MSE-R) and the simple adjustment to AVMSE (MSE-AV) provide quite satisfactory estimates for this configuration at all mode numbers, as might be expected from the estimates of error shown in Fig. 5(a). It can be inferred from Fig. 4(a) and confirmed by Fig. 7 that all modifications to MSE considered provide better predictions of the mode number (i.e. stiffness ratio) at which the maximum system loss factor is achieved. As was also seen from the computations for the prototype system, the lesser of the MSE and AVMSE provides an upper bound on the loss factor, while either MSE-R or the simple adjustment (Simple) provides a lower bound.

Because the solution for the plate and constraining layer with simply supported edges and no in-plane loads is equivalent to that of the similarly supported beam system after the substitution of Eq. (30), we may also compare the predictions resulting from MSE and the modifications with the predictions of the solution that is exact, to within the classic assumptions of the RUK analysis. Such a comparison is shown in Fig. 8 for a simply supported square plate of side length $177.8 \mathrm{~mm}$ (7 in.) and thickness $1.524 \mathrm{~mm}(0.060 \mathrm{in})$ with a thin $(0.127 \mathrm{~mm}$ or $0.005 \mathrm{in})$ constraining layer, both of modulus $E=69 \mathrm{GPa}$ or $10 \mathrm{Mpsi}, v=0.33$, and $\rho=0.1 \mathrm{lb} / \mathrm{in}^{3}(2.77 \mathrm{gm} / \mathrm{cc})$. The shear layer properties are $G_{R}=2.1 \mathrm{MPa}$ or $300 \mathrm{psi}, v=0.499, \rho=0.035 \mathrm{lb} / \mathrm{in}^{3}(0.97 \mathrm{gm} / \mathrm{cc})$, and $\eta=1$. For these parameters, the coupling coefficient, $Y$, of Eq. (26) is 0.36 .

Agreement between the analytic (extended RUK) solution and the FEA result is seen to be best for modes having nodal lines in only one direction, i.e., the 1st, 2nd, 4th, 6th and 10th modes, suggesting that the simplifying 


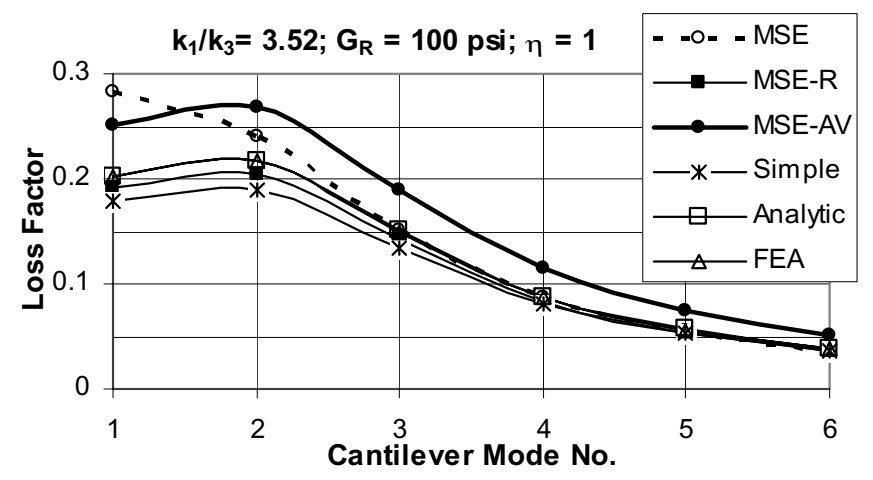

Fig. 7. System loss factors for symmetric sandwich beam, several methods.

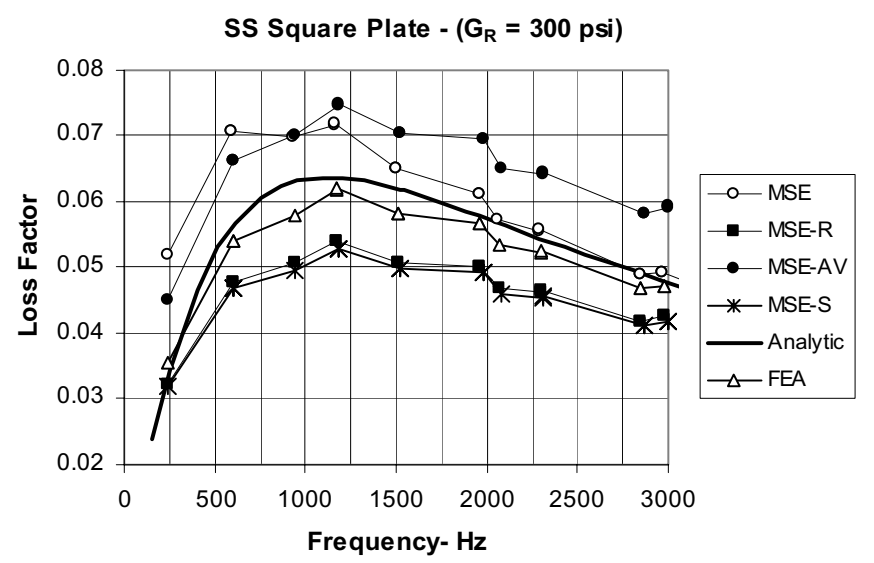

Fig. 8. System loss factors for damped square plate, several methods.

assumptions of the deformation field are most successful for the less complex mode shapes. Results from MSE and all modifications reflect the same departures form the smooth trend predicted by the extended RUK analysis. Once again, the use of the absolute value of the modulus with MSE is seen to lead to better predictions for the lower frequency (long wavelength and high stiffness ratio) modes, and poorer results for the modes with shorter wavelengths and low stiffness ratios. This is to be expected from Fig. 5(b), the computations for which correspond to the same value of $Y=k_{1} / k_{3}$. As also may be inferred from Fig. 5(b), the advantages of the Rongong and simple modifications to the method of MSE are less advantageous in the case of damping treatments with thin constraining layers than in the case of sandwich configurations. But once again, the lesser of the MSE and AVMSE (MSE-AV) is seen to provide an upper bound on the loss factor, while either MSE-R or the simple (Simple) adjustment is seen to provide a lower bound.

Because the modulus of viscoelastic materials is highly frequency dependent, one must consider that the shear layer in the results of Figs 7 and 8 consisted of a different viscoelastic material for each mode. A single frequencydependent material would not necessarily show the same trends in the change in modal damping with increasing mode number. However, the comparison of results from different methods for the same mode remains appropriate.

\section{Summary and conclusions}

The application of the method of Modal Strain Energy has been found to lead to over-predictions of the system loss factor. By focusing on a single degree-of-freedom system for which an exact solution is easily obtained, we have isolated the error resulting from treating the dissipative component as elastic in the determination of displacements and energies. 
Expressions for the complex-valued stiffness of the prototype four-parameter system were developed. In the case of a damping element with a very low stiffness, the traditional application of the MSE method was found to be appropriate. For systems with higher damping element stiffness, however, the error resulting from the use of MSE was seen to be come significant unless the material loss factor of the damping element is small (i.e., $\ll 1$ ). Several modifications to MSE were considered, and each was found to provide improvements in systems where the damping element(s) have loss factors of order unity and a high stiffness. Moreover, each of these methodologies was found to have the advantage of better predicting the configuration for maximum damping and are therefore useful improvements in the use of the method of MSE for design purposes. While comparisons were made only for the case where the material loss factor of the damping material had a loss factor of unity, such values are characteristic of the materials in general use in constrained layer damping treatments.

Of all modifications considered, Rongong's modification was found to provide the smallest error, particularly in the case of sandwich configurations. As this method leads to a lower bound on the loss factor, it enables the development of a conservative design. In this context, it is useful to note that one may use the lesser of the loss factors predicted by traditional Modal Strain Energy (MSE) and the modification obtained through the use of the absolute value of the modulus (AVMSE) to obtain an upper bound on the system loss factor.

Comparisons made on the basis of the prototype four-parameter system were confirmed by applying each methodology to configurations for which an exact analytic solution is available for extensions to the classical analysis by Ross, Ungar, and Kerwin. Results for a damped sandwich cantilever beam and for a simply supported square plate with a thin constraining layer supported the general conclusions drawn from the analysis of the prototype system.

\section{Acknowledgements}

The authors are indebted to Dr. Charles Cross, Air Force Research Laboratory, and to Dr. Jack Henderson, Universal Technology Incorporated, for their encouragement in this effort, and to Mr. Robert Gordon, Air Force Research Laboratory for providing useful discussions and computer codes for the FEA analyses used in this study, and to the Air Force Institute of Technology for providing computational resources.

\section{References}

[1] C.D. Johnson and D.A. Kienholz, Finite Element Predictions of Damping in Structures with Constrained Viscoelastic Layers, AIAA Journal 20(9) (Sept. 1982), 1284-1290.

[2] E.E. Ungar and E.M. Kerwin, Jr, Loss Factors of Viscoelastic Systems in Terms of Energy Concepts, Journal of the Acoustical Society of America 34(7) (1962), 954-957.

[3] D.R. Morgenthaler, The Absolute Value Modal strain Energy Method, Proceedings of Damping '91, WL-TR-3078, Vol. II, Wright Laboratory Flight Dynamics Directorate, August 1991, FDB-1-16.

[4] J.A. Rongong, Reducing Vibration Levels using 'Smart Joint' Concepts, Proceedings of IMSA 25, Noise and Vibration Engineering, Leuven, Belgium, 2000, 817-824.

[5] J.G. McDaniel and J.H. Ginsberg, Fundamental Tests of Two Modal Strain Energy Methods, Journal of Vibration and Acoustics, ASME 118 (April 1996), 272-275.

[6] C. Zener, Elasticity and Anelasticity of Solids, University of Chicago Press, Chicago, 1948.

[7] E.R. Marsh and L.C. Hale, Damping of Flexural Waves with Imbedded Viscoelastic Materials, Journal of Vibration and Acoustics, ASME 120(1) (January 1988), 188-193.

[8] R.M. Christensen, Theory of Viscoelasticity, 2nd ed., Academic Press, New York, 1982.

[9] A.D. Nashif, D.I.G. Jones and J.P. Henderson, Vibration Damping, John Wiley \& Sons, New York, 1985.

[10] P.J. Torvik and B.D. Runyon, Observations on the Accuracy of Finite Element Predictions of Constrained Layer Damping, Proceedings of the 10th National Turbine Engine High Cycle Fatigue (HCF) Conference, New Orleans, March 8-11, 2005.

[11] P.J. Torvik and B.D. Runyon, On the Application of the Method of Modal Strain Energy to the Determination of Loss Factors for Damped Sandwich Beams, Proceedings, 75th Shock and Vibration Symposium, Virginia Beach, VA, October 17-21, 2004.

[12] D. Ross, E.E. Ungar and E.M. Kerwin, Jr, Damping of Plate Flexural Vibrations by Means of Viscoelastic Laminae, in: Structural Damping, J.E. Ruzicka, ed., ASME, NY, 1959, pp. 49-88.

[13] D.K. Rao, Vibration of Short Sandwich Beams, Journal of Sound and Vibration 52(2) (1977), $253-263$.

[14] F. Abdulhadi, Transverse Vibrations of Laminated Plates with Viscoelastic Damping, Shock and Vibration Bulletin 40(Part 5) (1969), 93-104. 
[15] P.J. Torvik and B.D. Runyon, On Estimating the Loss Factors of Plates with Constrained Layer Damping Treatments, AIAA-2006-2235, 75th AIAA/ASME/AHS Structures, Structural Dynamics and Materials Conference, Newport, R.I., May 1-4, 2006. (Also submitted for publication.)

[16] D.I.G. Jones, Handbook of Viscoelastic Vibration Damping, John Wiley \& Sons, Ltd., Chichester, 287-288.

[17] R. Plunkett and C.T. Lee, Length Optimization for Constrained Layer Damping, Journal of the Acoustical Society of America 48(No. 1, Part 2) (July 1970), 150-161

[18] N.L. Soni and F.K. Bogner, Finite Element Analysis of Viscoelastically Damped Sandwich Structures, AIAA Journal 20(5) (1982), $700-707$.

[19] C.T. Sun, B.V. Sankar and V.S. Rao, Damping and Vibration Control of Unidirectional Composite Laminates using Add-on Viscoelastic Materials, Journal of Sound and Vibration 139(2) (1990), 277-287. 

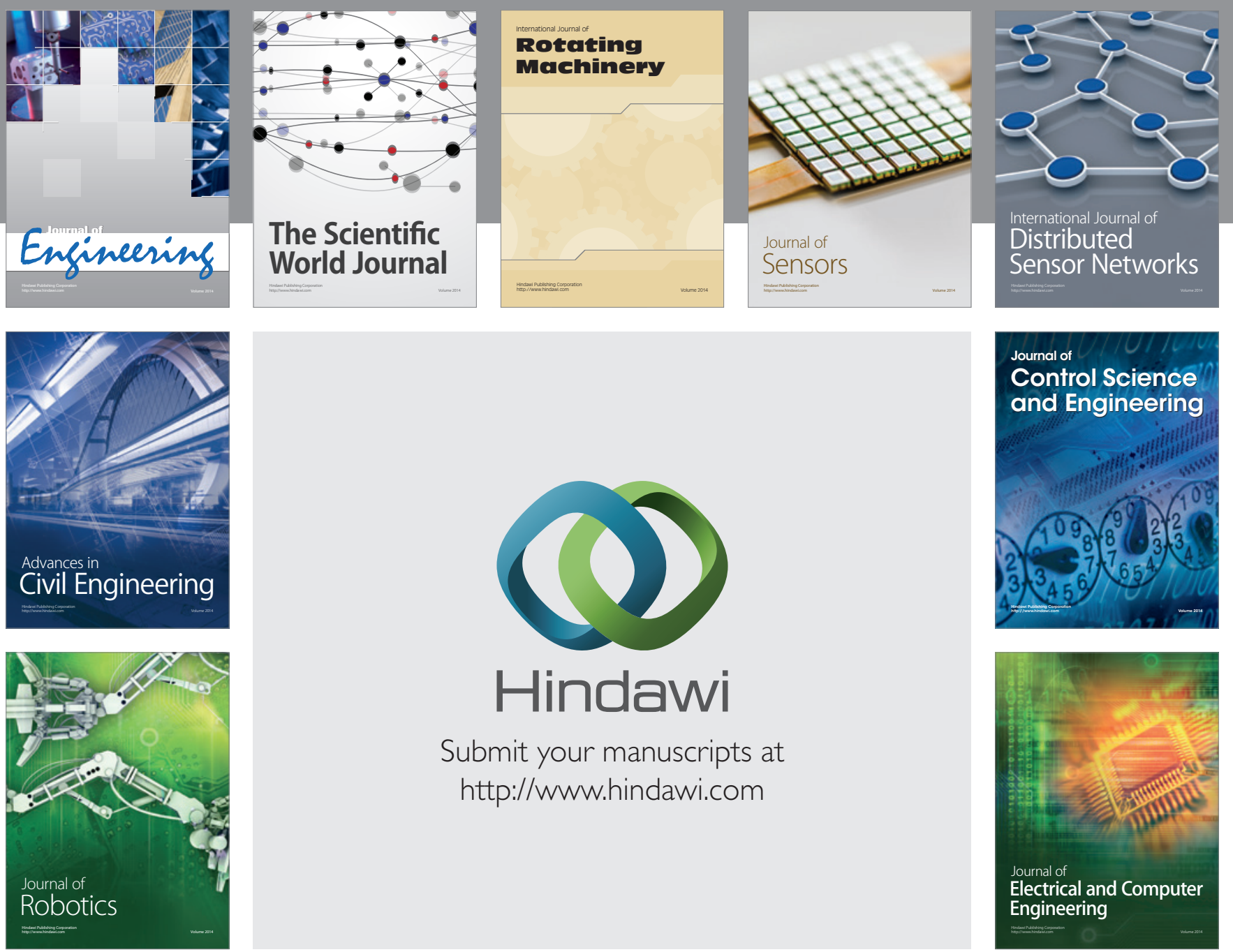

Submit your manuscripts at

http://www.hindawi.com
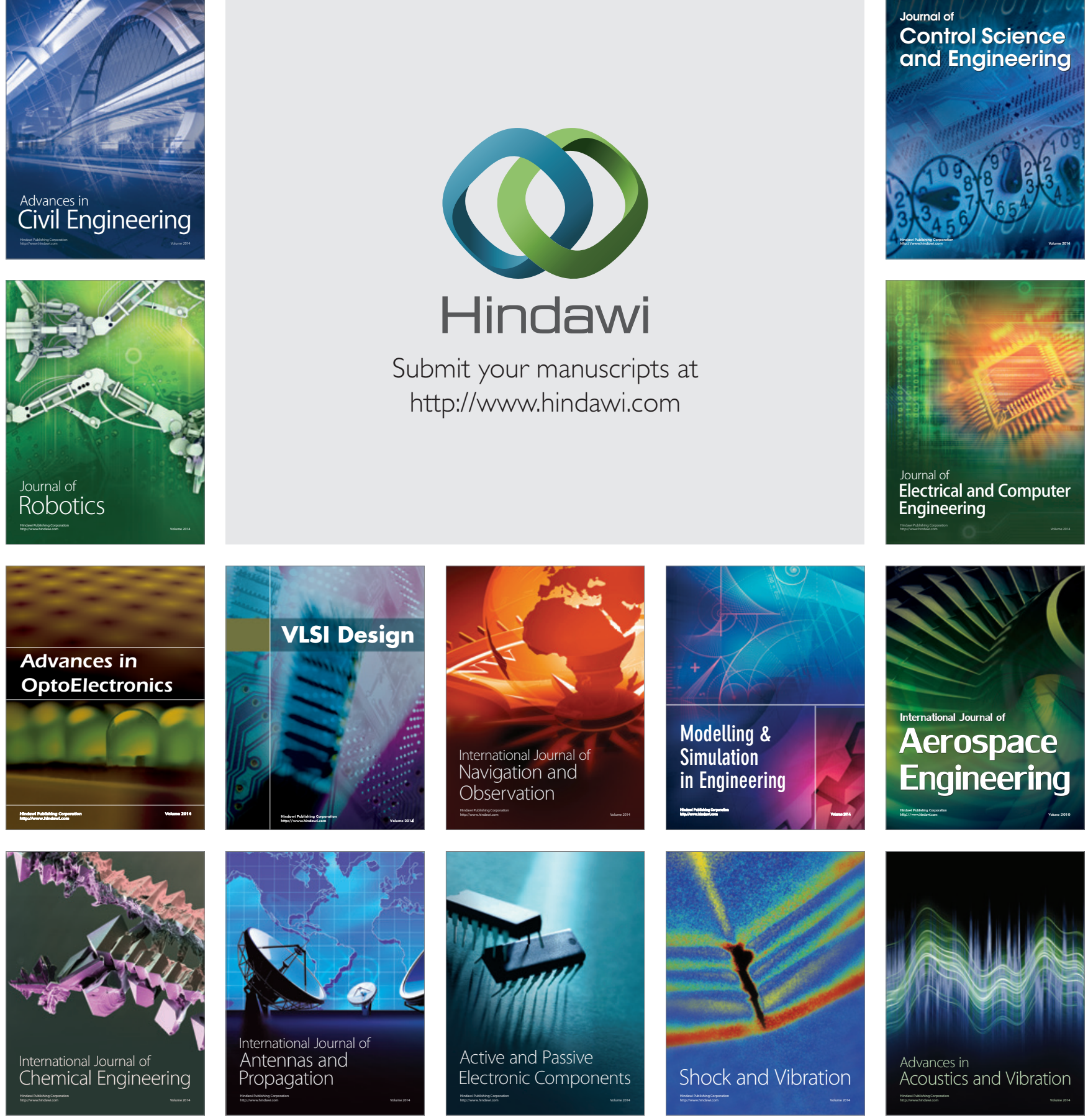